\title{
Correction To: Finite Indentation of Pressurized Elastic Fluid Nanovesicles by a Rigid Cylindrical Indenter ${ }^{\star \star}$
}

\author{
Xingyi Tang ${ }^{1} \quad$ Jianxiang Wang ${ }^{1,2} \quad \mathbf{X i n}_{\mathbf{Y i}^{1,3 \star}}$ \\ $\left({ }^{1}\right.$ Department of Mechanics and Engineering Science, College of Engineering, Peking University, Beijing \\ 100871, China) \\ $\left({ }^{2}\right.$ CAPT, HEDPS and IFSA Collaborative Innovation Center of MoE, Peking University, Beijing 100871, \\ China) \\ $\left({ }^{3}\right.$ Beijing Innovation Center for Engineering Science and Advanced Technology, Peking University, Beijing \\ 100871, China)
}

published online 24 July 2019

(C) The Chinese Society of Theoretical and Applied Mechanics 2019

\section{Correction To: Acta Mechanica Solida Sinica https://doi.org/10.1007/s10338-019-00107-5}

Unfortunately due to a typesetting mistake in the caption of Fig. 1 , fourth line, $l_{2}$ has been displayed wrongly as $I_{2}$.

The original article has been corrected.

\footnotetext{
* Corresponding author. E-mail: xyi@pku.edu.cn

** The original article can be found online at https://doi.org/10.1007/s10338-019-00107-5.
} 